\title{
Examining the diagnostic pathway for lung cancer patients in Wales using discrete event simulation
}

\author{
Tracey J. England ${ }^{1}$, Paul R. Harper ${ }^{1}$, Tom Crosby ${ }^{2}$, Daniel Gartner ${ }^{1}$, Edilson F. Arruda ${ }^{1,3}$, \\ Kieran G. Foley ${ }^{2}$, Ian J. Williamson ${ }^{4}$ \\ ${ }^{1}$ School of Mathematics, Cardiff University, Cardiff, UK; ${ }^{2}$ Velindre Cancer Centre, Cardiff, UK; ${ }^{3}$ Alberto Luiz Coimbra Institute-Graduate School and \\ Research in Engineering, Federal University of Rio de Janeiro, Rio de Janeiro, RJ 21941-972, Brazil; ${ }^{4}$ Aneurin Bevan University Health Board, Newport, UK \\ Contributions: (I) Conception and design: TJ England; (II) Administrative support: None; (III) Provision of study materials or patients: None; (IV) \\ Collection and assembly of data: None; (V) Data analysis and interpretation: TJ England; (VI) Manuscript writing: All authors; (VII) Final approval \\ of manuscript: All authors. \\ Correspondence to: Dr. Tracey J. England. School of Mathematics, Cardiff University, Senghennydd Road, Cardiff, CF24 4AG, UK. \\ Email: EnglandTJ@cardiff.ac.uk; T.J.England@soton.ac.uk.
}

\begin{abstract}
Background: UK's National Health Service (NHS) has one of the poorest lung cancer survival rates in Europe. To improve patient outcomes, a single cancer pathway was introduced in the NHS. In this study, a Discrete Event Simulation was developed to understand bottlenecks during lung cancer treatment.

Methods: This study focused on the lung cancer diagnostic pathways at two Welsh hospitals. Discrete Event Simulation is a computer-based method that has been effectively used in demand and capacity planning. In this study, simulation models were developed for the current and proposed single cancer pathways. The validated models were used to provide Key Performance Indicators. Several "what-if” scenarios were considered for the current and proposed pathways.

Results: Under the current diagnostic pathway, the mean time to treatment for a surgery patient was 68 days at the Royal Glamorgan Hospital and 79 days at Prince Charles Hospital. For chemotherapy patients, the mean time to treatment was 52 days at the Royal Glamorgan Hospital and 57 days at Prince Charles Hospital. For radiotherapy patients, the mean time to treatment was 44 days at Royal Glamorgan Hospital and 54 days at Prince Charles Hospital. Ensuring that the patient attends their first outpatient appointment within 7 days and streamlining the diagnostic tests would have the potential to remove approximately 20 days from the current lung cancer pathway resulting in a 20-25\% increase of patients receiving treatment within 62 days. Ensuring that patients begin their treatment within 21 days of diagnosis sees almost all patients comply with the 62-day target.

Conclusions: Discrete Event Simulation coupled with a detailed statistical analysis provides a useful decision support tool which can be used to examine the current and proposed lung cancer pathways in terms of time spent on the pathway.
\end{abstract}

Keywords: Lung cancer; diagnostics; simulation models; optimal pathway

Submitted Sep 03, 2020. Accepted for publication Dec 23, 2020.

doi: $10.21037 /$ tlcr-20-919

View this article at: http://dx.doi.org/10.21037/tlcr-20-919

\section{Introduction}

Lung cancer is the third most common cancer in the UK (accounting for $13 \%$ of all new cancer cases) and the leading cause of cancer mortality (1). Poor lung cancer outcomes are partly due to delays in timely diagnosis (1).
Approximately two thirds of patients diagnosed with lung cancer begin their treatment within the current 62-day target (2), with many being diagnosed with advanced stage of disease. Delays are thought to be due to, in part, an increase in the number of urgent referrals, as well as patients following more personalised complex pathways 
with a wider selection of diagnostic tests available (2). There is also a lack of diagnostic workforce and infrastructure. The UK Lung Cancer Coalition (UKLCC) report " 25 by 25 " found that $65 \%$ of health specialists dealing with lung cancer believe that early diagnosis is the most important factor in improving survival rates (3).

While patient experience is good, lung cancer survival in Wales is poor because of late stage diagnosis and treatment. Only $14.5 \%$ of Welsh lung cancer patients are still alive after 5 years (4) compared with 16.2\% in England, $17.3 \%$ in France and the Netherlands and 18.2\% in Belgium (5).

The Welsh Government (6) has recently set out its approach to improving cancer services and outcomes with a significant focus on the earlier detection of cancers and greater understanding and efficiency of the pathways that patients take. One result of this is the introduction of a single cancer pathway (SCP) (7). The SCP is the new target within Wales for diagnosing cancer and getting treatment started within 62 days of the date of suspicion of their cancer. The new pathway is designed to replace the previous two-tier pathway (for urgent and non-urgent referrals) and aims to reduce waiting times and improve early diagnosis. The National Optimal Lung Cancer Pathway (NOLCP) was adopted in Wales in August 2017 as a way of streamlining the diagnostic pathway and aims to reduce the time from referral to treatment from 62 days to 49 days (2).

Timely diagnostic testing for suspected cancers is critical and requires the effective and efficient deployment of resources. Underpinning this is the necessity to analyse demand and capacity. The overall aim behind this research was to align capacity to best match demand, and to ultimately improve patient care and outcomes. One difficulty in estimating the demand and capacity is the variation in the system; no two patients are the same. Whilst mathematical capacity planning methods that take account of variation are available (8), average based estimates which underestimate capacity requirements are often used $(9,10)$.

Discrete event simulation (DES) is a computer-based method that has been an effective tool for demand and capacity planning (11). DES allows the modelling of complex systems at an individual patient level. The systems modelled can be thought of as queuing networks where individual entities pass through a series of discrete events, one by one, at discrete intervals and wait in queues if the resource for a given event is limited. DES also allows "whatif" scenario analysis which, in turn, enables us to assess the possible impact of different policy or resource decisions.

DES has been successfully used to inform health service planning projects for breast cancer (12), colorectal cancer (13), chlamydia (14), HIV (15), emergency medical services (16), out of hours services (17) and trauma and orthopaedic services (18).

Whilst DES has been used in cancer screening programmes $(12,13)$, there is little literature to suggest that it has been widely used in modelling the cancer diagnosis pathway. DES modelling was suggested as an approach for representing the lung cancer diagnosis pathway in a recent paper by Ju and colleagues (19). However, the implementation of the model is not presented. One possible reason for this and for the lack of other peer-reviewed studies is the complexity of the pathway and the variation associated with each patient's journey.

Our study therefore provides an example of how simulation can be used to model both a current and a proposed diagnostic pathway for lung cancer. In this research we demonstrated how DES models can be used to represent the diagnostic pathway for lung cancer patients in Wales and evaluated potential reductions of the time until the decision to treat with the introduction of the proposed single cancer pathway. The aim of the study supports the aim of the Single Cancer Pathway (SCP) which is to identify areas where gains can be made so that a patient's time to diagnosis and subsequent treatment can be reduced, thus improving the patient's outcome. Whilst this study focused on two hospitals in Wales, it is envisaged that the findings can prove beneficial for other locations within the UK and internationally.

We present the article in accordance with the STRESS reporting checklist for discrete event simulation modelling (20) (available at http://dx.doi.org/10.21037/tlcr20-919).

\section{Methods}

This study focuses on the lung cancer diagnostic pathways at two hospital sites within Cwm Taf Morgannwg University Health Board (CTMUHB); Royal Glamorgan Hospital in Llantrisant and Prince Charles Hospital in Merthyr Tydfil. CTMUHB provides primary, community, hospital, and mental health services to 450,000 people living in Bridgend, Merthyr Tydfil and Rhondda Cynon Taf.

CTMUHB was chosen to participate in this study as its cancer services have implemented Tracker 7 software into their patient administrative systems (PAS), to track patients prospectively through their cancer pathway (21). Other sites in the UK would link datasets from their PAS 
Table 1 Total number of suspected lung cancer referrals by USC/nUSC and status (Jan 2018 - Nov 2019)

\begin{tabular}{lccccccc}
\hline Pathway & Total treated & $\%$ & Total downgraded & $\%$ & Other & $\%$ & Total \\
\hline Prince Charles Hospital (Urgent Suspected Cancer) & 86 & 26.71 & 234 & 72.67 & 2 & 0.62 & 322 \\
Prince Charles Hospital (Non-Urgent Suspected Cancer) & 181 & 50.84 & 170 & 47.75 & 5 & 1.40 & 356 \\
Royal Glamorgan Hospital Urgent Suspected Cancer & 95 & 32.87 & 192 & 66.44 & 2 & 0.69 & 289 \\
Royal Glamorgan Hospital Non-Urgent Suspected Cancer & 152 & 64.96 & 79 & 33.76 & 3 & 1.28 & 234 \\
\hline
\end{tabular}

USC, urgent suspected cancers; nUSC, non-urgent suspected cancers.

systems with those from pathology and imaging to obtain the required information for the model. This paper focuses on the simulation models developed for the lung cancer services at Prince Charles and Royal Glamorgan Hospitals in CTMUHB.

The study did not require ethical approval as no patients were directly involved and the data used to determine the model parameters was fully anonymized prior to use.

\section{Data source}

A thorough analysis of the data collected through the Tracker 7 software was conducted. The data contains 1,201 records for patients referred to either Royal Glamorgan Hospital or Prince Charles Hospital with suspected lung cancer. The data also contains the dates associated with 1,928 diagnostic tests carried out for these patients. Each row represented a detailed patient record including their longitudinal history of diagnostic tests (dates of referral, dates undertaken, reporting times etc.) between January 2018 and November 2019.

The referral data also included the dates of their first outpatient appointment, Multi-Disciplinary Team (MDT) meetings, and their decision to treat date. The first treatment that the patient received was also included. Knowing the initial treatment plan allows investigation of whether patients with different treatments spend different lengths of time in the system.

\section{Statistical analysis}

The referral data was analysed to determine the number of referrals at each hospital as well as the inter-arrival time between successive patients. The referrals were subdivided according to whether they were an Urgent Suspected Cancer (USC) or a non-Urgent Suspected Cancer (nUSC). The total number of referrals, broken down by pathways (USC and nUSC) and status (treated/downgraded/other) for each hospital are provided in Table 1 .

Stat-Fit for Simul8 and Easy Fit 5.6 Professional were used to fit the probability distributions to the inter-arrival times, for each of the pathways, at both hospitals. The inter-arrival time distributions for the USC pathways were Gamma $(0.8841,2.5576)$ for Prince Charles Hospital and Gamma $(0.85171,2.8374)$ for Royal Glamorgan Hospital. The corresponding distributions for the nUSC pathways were determined as Gamma $(0.89564,2.1796)$ and Gamma $(0.78502,3.7833)$.

Table 2 features the number of diagnostic tests carried out at each hospital. It is interesting to note that Prince Charles Hospital (PCH) performed approximately 300 more diagnostic tests than Royal Glamorgan Hospital (RGH) over the same time-period; with more CTs and PET-CTs (conducted externally) undertaken. The Tracker 7 data also shows that for patients undergoing surgery, radiotherapy or SACT, the time until treatment is slightly longer at PCH than at RGH. It is also worth highlighting that while there are 78 PET-CT scanners in the UK (2), there is only one in South Wales and it is shared between several health boards in the area and used across all cancer sites.

Further analysis of the diagnostic test data on Tracker 7 shows that $81 \%$ of patients ( 691 of 851 ) have 3 or fewer tests (Table 3) with the first test most likely to be a CT scan. For patients treated with curative intent, the most likely diagnostic pathway was an initial CT followed by a PETCT and biopsy.

Considering the diagnostic tests, we define:

* Time to arrange: the time between the request date and the date when the test was carried out.

* Reporting time: the time between the test taking place and the report being produced.

* Total time: the sum of the Time to arrange and Reporting time.

The time to arrange and the reporting time for each patient having a particular diagnostic test are used to derive the probability distributions used in the simulation 
Table 2 Number of diagnostic tests at PCH and RGH (Jan 2018 - Nov 2019)

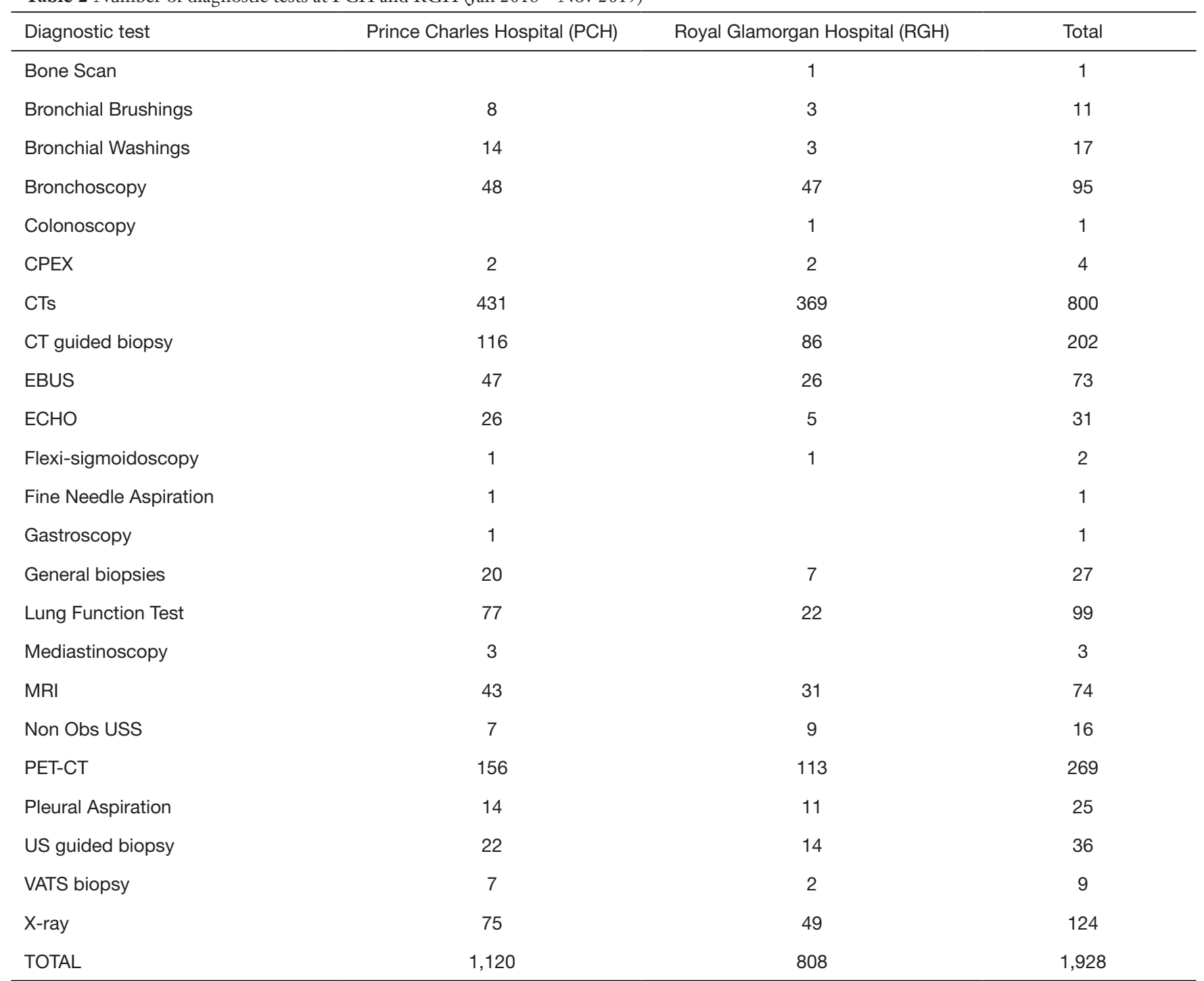

CPEX, cardio-pulmonary exercise testing; CTs, computerised tomography including abdomen, thorax, head, colon, tap, urinary tract; CT, computerised tomography; EBUS, endobronchial ultrasound scan; ECHO, echocardiogram; MRI, magnetic resonance imaging; non Obs USS, non-obstetric ultrasound scan; PET-CT positron emission tomography-computed tomography; US, ultrasound; VATS, video-assisted thoracoscopic surgery.

model for a given test at each hospital site. Stat-Fit for Simul8 and Easy Fit 5.6 Professional were used to fit the statistical distributions. A full list of the fitted parameters is provided. For example, the time to arrange a CT scan at Prince Charles Hospital is represented as a Normal (6.6149, 17.806) distribution. The time to report a given diagnostic is further divided into two-time intervals: the time needed for a radiologist to complete their other tasks prior to carrying out their reporting duties and the time needed to produce the report. In the Prince Charles Hospital model, the time to complete other work prior to the CT report is represented as a Gamma $(0.9842,9.1687)$ distribution and the time to report as an Exponential (0.01388889) distribution. The corresponding distributions for the Royal Glamorgan Hospital are similar with the time to complete prior work represented as a Gamma $(0.96864,8.1805)$ and the time to report a CT scan also represented as an Exponential (0.01388889) distribution. 
Table 3 Number of diagnostic tests per patient (Jan 2018 - Nov 2019)

\begin{tabular}{lcc}
\hline Number of tests & Number of patients & No of tests \\
\hline 1 & 347 & 347 \\
2 & 188 & 376 \\
3 & 156 & 468 \\
4 & 89 & 356 \\
5 & 43 & 215 \\
6 & 18 & 108 \\
7 & 7 & 49 \\
8 & 2 & 16 \\
9 & 1 & 9 \\
Total & 851 & 1,944 \\
\hline
\end{tabular}

The average times associated with the most prevalent tests used at each hospital are shown in Tables 4 (Prince Charles Hospital) and 5 (Royal Glamorgan Hospital) while the full list of model parameters is provided separately.

Tables 4,5 shows that the largest delays are associated with the Endobronchial Ultrasound (EBUS) sampling of lymph nodes used for both diagnosis and staging in both hospitals. Previously, patients were referred to an external health board for EBUS. Recently, the Cwm Taf Morgannwg University Health Board (CTMUHB) has purchased its own EBUS facility, which suggests that delays due to this specific diagnostic test should be reduced in the future.

The Tracker 7 data for each of the diagnostic tests listed in Tables 4,5 has been used to parameterise the models for each hospital.

In particular, the model uses the inter-arrival rates, and the service times for each clinic appointment and diagnostic test carried out. As well as the parameters estimated from the referral and diagnostic data, expert opinion was elicited from the lung consultants and radiologists at both hospitals to provide other parameter estimates needed in the model. For example, the time needed for a radiologist to vet a scan request or to produce a specific report. In total, each model requires approximately 50 parameters to describe the diagnostic pathway for a lung cancer patient attending either of the two hospital sites.

\section{Model}

We have developed detailed computer simulation models (using SIMUL8 software) to capture the diagnostic pathway for each hospital site.

\section{Model structure}

In addition to the model of the current diagnostic pathway (Figure 1), we also developed a corresponding model for the single cancer pathway (SCP) (Figure 2). This allowed us to examine the benefits of introducing the new pathway in terms of the time to diagnosis. The aim of the National Optimal Lung Cancer Pathway is to reduce the time to diagnosis to 28 days. Each model allowed us to simulate individual patients on their diagnostic pathway. The sequence of tests in the current lung cancer pathway simulation model is based on the recommendations of respiratory consultants at each hospital site. As the current lung cancer pathways are implemented slightly differently at each hospital, there are two simulation models, one for each hospital and each with its own set of parameters. For example, Tables 4,5 show that the time to arrange and report each diagnostic test differ across hospital sites and it is important that the models reflect these differences.

Under the current lung cancer pathway at both hospitals (Figure 1), a patient is referred and then triaged. Patients are then sent for a CT scan and the results are discussed at a diagnostic multi-disciplinary team (MDT) meeting before the patient is seen in an outpatient clinic. Patients suitable for radical curative treatment (e.g., surgery, chemotherapy, or radiotherapy) are referred for further combinations of diagnostic tests, usually starting with a PET-CT and then a biopsy (CT-guided or US guided), EBUS or bronchoscopy depending on radiological findings. An MRI may be requested to confirm equivocal findings on former tests. All the results will be discussed at a second MDT meeting to agree a treatment recommendation. The patient will then attend a further clinic appointment and their treatment options will be discussed and management plan agreed. The simulation model for the current lung cancer pathway considers the patient's pathway from their point of referral to the time that their treatment starts.

With the optimal lung cancer pathway (Figure 2), a patient is referred and then triaged before being sent for a CT thorax abdomen pelvis (CT TAP). The patient then attends a Fast-Track Clinic before being referred for further diagnostic tests. As with the current pathway, patients receiving radical treatment will have a PET-CT scan and depending on results, further staging investigations and lung function tests may be required. Patients requiring chemotherapy will need to wait for the tumour genomic results before agreeing their treatment plan. Under the 
Table 4 Time associated with diagnostic tests carried out by Prince Charles Hospital (in days)

\begin{tabular}{lccc}
\hline Diagnostic test & Time to arrange & Reporting time & Total \\
\hline Bronchoscopy & 5.33 & $0.16^{*}$ & 5.49 \\
CTs & 6.27 & 4.56 & 10.83 \\
CT guided biopsy & 7.13 & 7.71 & 14.84 \\
EBUS & 19.98 & 8.14 & 28.12 \\
LFT & 13.55 & 0.08 & 13.63 \\
MRI & 9.93 & 4.37 & 14.3 \\
PET-CT & 10.74 & 0.91 & 11.65 \\
US guided biopsy & 10.55 & 7.35 & 17.9 \\
X-ray & 0.75 & 6.64 & 7.39 \\
\hline
\end{tabular}

*, pathology time not included. CTs, Computerised tomography including abdomen, thorax, head, colon, TAP, urinary tract; CT, computerised tomography; EBUS, endobronchial ultrasound scan; LFT, lung function test; MRI, magnetic resonance imaging; PET-CT, positron emission tomography-computed tomography; US, ultrasound.

Table 5 Time associated with diagnostic tests carried out by Royal Glamorgan Hospital (in days)

\begin{tabular}{lccc}
\hline Diagnostic test & Time to arrange & Reporting time & Total \\
\hline Bronchoscopy & 4.40 & 4.24 & 8.64 \\
CTs & 6.53 & 4.13 & 10.66 \\
CT guided biopsy & 6.06 & 7.57 & 13.63 \\
EBUS & 18.96 & 10.00 & 28.96 \\
LFT & 2.59 & 0.86 & 3.45 \\
MRI & 2.47 & 1.52 & 3.99 \\
PET-CT & 14.64 & 0.58 & 15.22 \\
US guided biopsy & 6.36 & 7.73 & 14.09 \\
X-ray & 0.94 & 3.19 & 4.13 \\
\hline
\end{tabular}

CTs, computerised tomography including abdomen, thorax, head, colon, TAP, urinary tract; CT, computerised tomography; EBUS, endobronchial ultrasound scan; LFT, lung function test; MRI magnetic resonance imaging; PET-CT, positron emission tomographycomputed tomography; US, ultrasound.

optimal cancer pathway, there should be no more than 3 days between the MDT and the decision to treat (DTT). as with the current lung cancer pathway, the single cancer pathway (SCP) simulation model separates the treatment options. The simulation model for the SCP discussed in this study represents the patient's journey from the point of suspicion until the decision to treat.

\section{Model outputs}

Each model monitored patient progress along the pathway. The model captured the average time in the system and the percentage of patients that are within a certain time threshold. In the current lung cancer pathway model, the time in the system represents the time between the point of referral (POR) by the general practitioner and the time of the first treatment. The target is 62 days. In the single cancer pathway, the time in the system represents the time between the point of suspicion of cancer (POS) and the Decision to Treat (DTT) which should be at most 28 days.

As the pathways are different for patients undergoing different treatment options, the results were captured for each of the main treatment categories (SACT, radiotherapy 


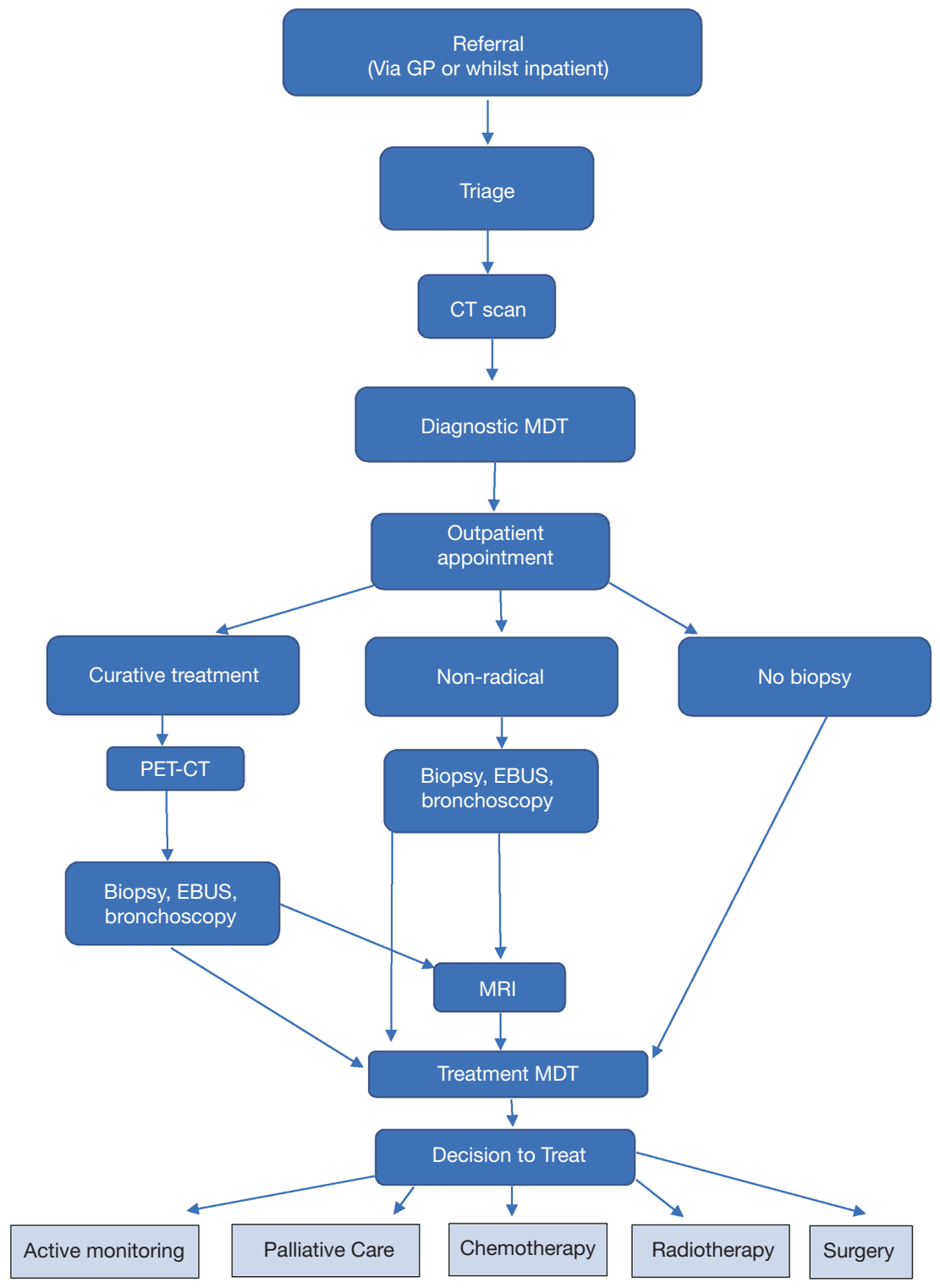

Figure 1 Current lung cancer pathway.

and surgery) along with palliative care and active monitoring. In the scenario analysis, the results for the SACT, radiotherapy and surgery pathways are presented and discussed.

\section{Model validation and verification}

Model validation and verification of the current pathway models ensures that each model sufficiently mimics the current lung cancer pathway implemented at each hospital site. The models for the proposed single cancer pathway (SCP) are compared with the schematic diagram described in the National Optimal Pathway for Lung Cancer document produced by the Wales Cancer Network and NHS Wales Health Collaborative in 2019 (7).

Scenario analyses were also conducted with both the current and SCP simulation models to aid future demand and capacity decisions. For example, scenarios considered the benefits associated with different levels of service provision for the diagnostic tests. Currently, in Wales, there are lengthy delays associated with certain diagnostic tests which can add two to three weeks to the patient's pathway (see Tables 4,5). This of course is not ideal given the aim to 


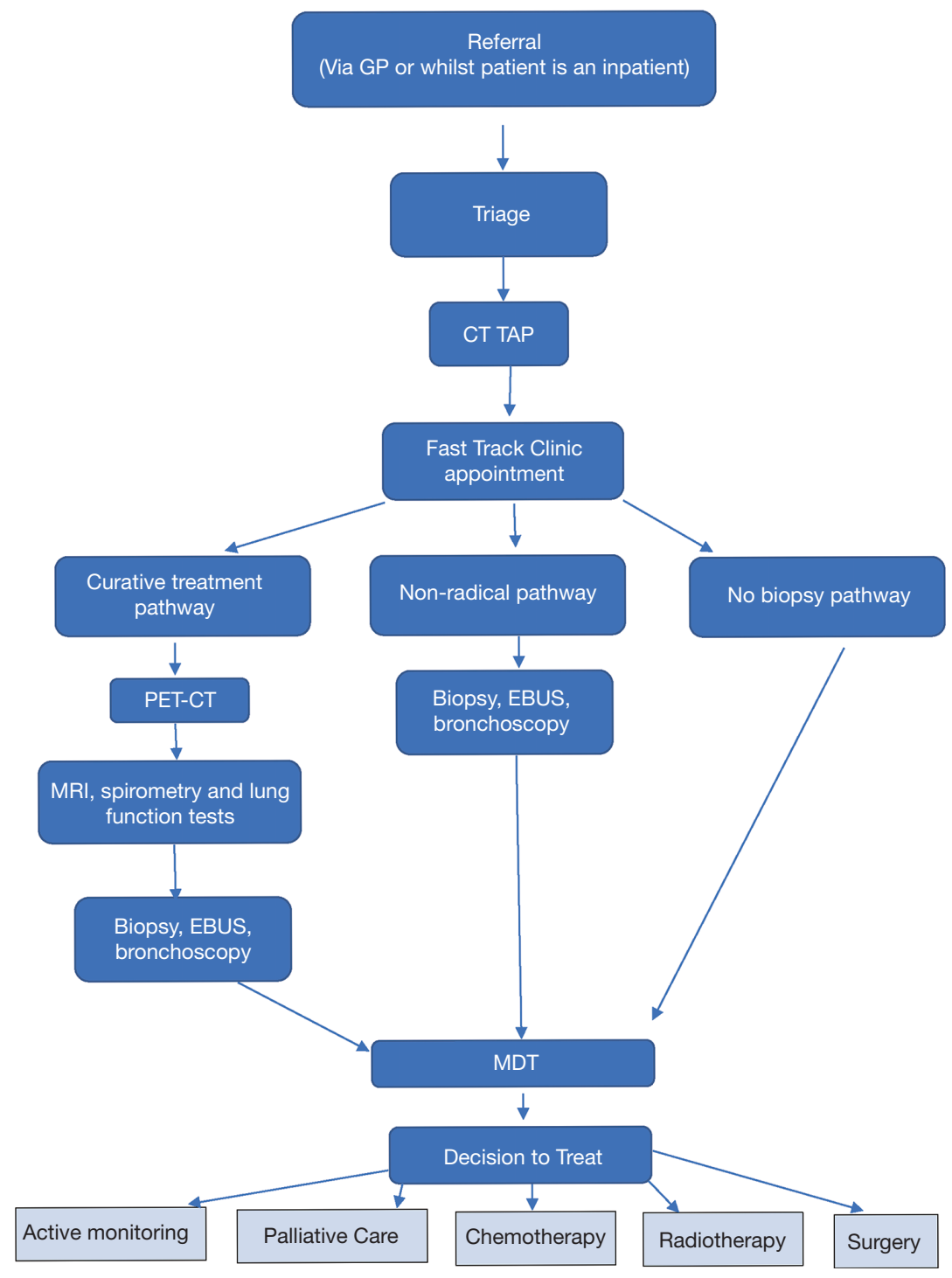

Figure 2 Single cancer pathway.

achieve a 62-day target for the start of treatment from the point of suspicion for $95 \%$ of the patients.

\section{Current lung cancer pathway model scenarios}

Eight scenarios were considered following discussions with the respiratory consultants involved in the study. The scenarios considered different levels of diagnostic test availability as well as reductions in the time to produce the necessary reports for a given test.

In the first scenario for the current lung cancer pathway model (Outpatients within 7 days) we considered the effect of all patients being seen in an outpatient clinic having had their initial CT scan within seven days of their date of suspicion.

In the next three scenarios, we considered the effect of different levels of service provision in the diagnostic tests. In the 7 days between tests scenario, we considered the effect of a weekly diagnostic service. In the 3 days between tests, we considered a bi-weekly service and in the 1 day between tests, a daily service.

The next two scenarios (tests reported within 2 days, tests reported within 1 day) consider the effect of reducing the time it takes to report a diagnostic test to 2 days or less. Tables 4,5 show that the current time to produce a 
report is more than 4 days for most of the diagnostic tests available.

The final two scenarios show a combination of strategies to reduce the time until treatment:

* Outpatients within 7 days, 3 days between tests and reported within 2 days: ensuring the patient is seen within 7 days of their date of suspicion and that tests are offered twice a week with the reports available within 2 days of the test.

* Outpatients within 7 days, 1 day between tests and next day report: ensuring the patient is seen within 7 days and that tests are offered daily with results available the next day.

Further scenarios were considered that examined the effect of reducing the time after a patient's case was discussed at an MDT and they started their treatment. The aim of the National Optimal Lung Cancer Pathway is that patients should begin their treatment within 21 days of their decision to treat. For example, one scenario considered the effect of reducing the time to treat by 10 days. For patients that undergo surgery this could mean that the patient is seen in an outpatient clinic within a few days of their MDT and have a shorter wait until their pre-assessment clinic prior to hospital admission. For radiotherapy and chemotherapy patients, they could also be seen in an appropriate outpatient clinic within a few days of their MDT and wait fewer days until their treatment is agreed and planned. Combining these scenarios with the ones discussed in this paper could potentially see all patients receive treatment within the 62-day target proposed by the Single Cancer Pathway.

Scenarios where the time to treatment was limited to 21 or 28 days were also considered.

\section{Single cancer patbway scenarios}

The baseline model for the single cancer pathway model considered the pathway implemented with the current level of reporting delay for each of the diagnostic tests at Prince Charles and Royal Glamorgan Hospitals. We also considered one "what-if" scenario.

Scenario 1 considered the effect of reducing the reporting delay so that diagnostic tests are reported within a day of the test.

The key performance indicators (mean time to diagnosis and $\%$ of patients that receive their diagnosis within 28 days) are presented in the Results section.

\section{Model evaluation}

Each of the simulation models was run for 300 iterations to ensure stable predictions of the key performance indicators (KPIs). The KPIs collected for the current lung cancer pathway were:

* The average time spent on the lung cancer pathway (with $95 \%$ confidence intervals)

* The percentage of patients that spent 62 days or less on the pathway (with $95 \%$ confidence intervals)

These were validated against the actual data. As well as the key performance indicators, the number of referrals, diagnostic tests, MDT meetings, outpatient clinics and the number of patients on each treatment pathway was recorded and compared with the data to validate the model. In each of the current pathway models, the model's accuracy in predicting these quantities was approximately $97 \%$, suggesting that the models were a very good representation of the current processes at each hospital.

The KPIs collected for the single cancer pathway were:

* The average time until diagnosis

* The percentage of patients that receive their diagnosis within 28 days

\section{Results}

The results for the baseline and scenario models for the current lung cancer pathway and the SCP at each hospital are presented.

\section{Current lung cancer patbway}

Table 6 shows the mean time to treatment on the current lung cancer pathway. The results have been separated according to the treatment that the patient is designated to receive.

The first scenario (outpatients within 7 days) considered the effect of ensuring that patients are seen in an outpatient clinic within 7 days of their date of suspicion. If this could be achieved, then between 10 and 12 days could be removed from the lung cancer pathway for all patients referred to Royal Glamorgan (RGH) and Prince Charles (PCH) Hospitals. For example, the mean time to diagnosis for surgery patients at the RGH would reduce from 68.15 to 57.55 days (see Table 6).

The second scenario, 7 days between tests, sees that providing a weekly diagnostic service provides a minor improvement with between one and four days removed 


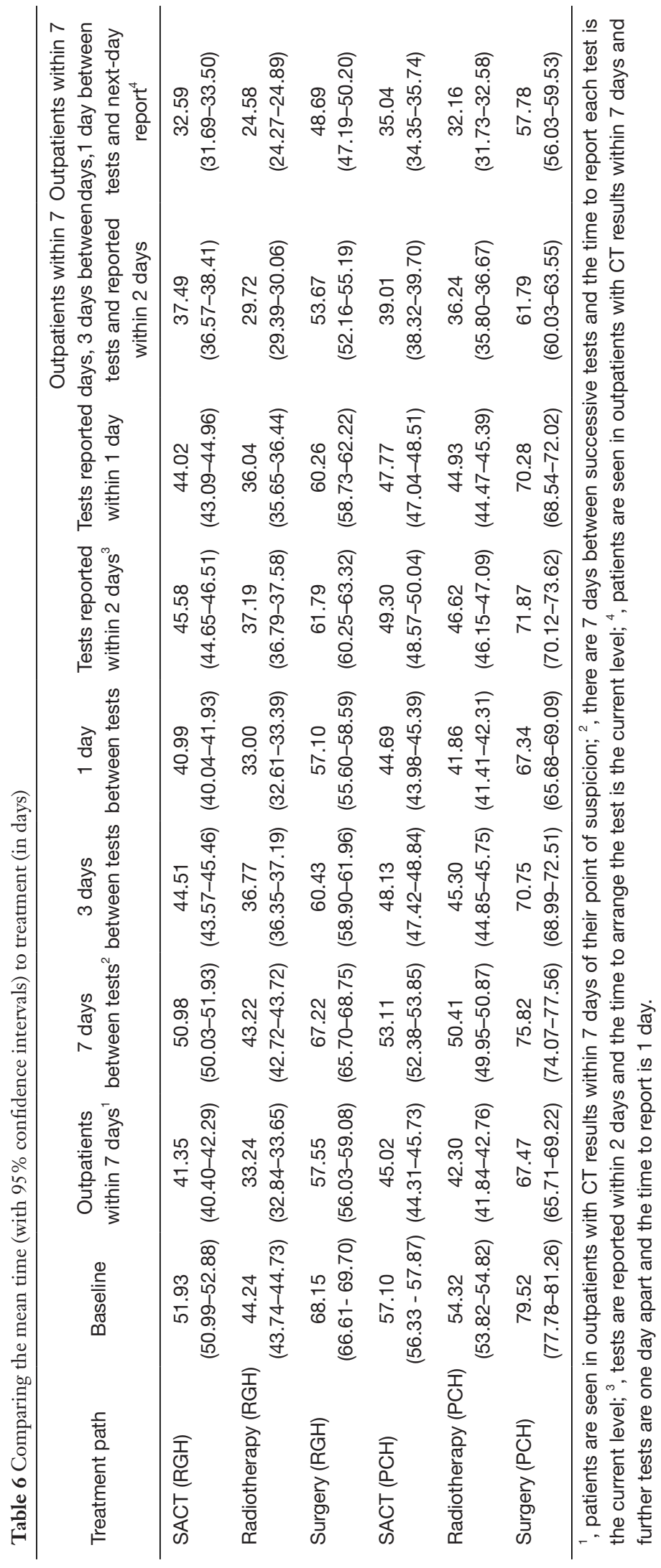


from the current pathway. However, a 10-day reduction can be achieved if a daily diagnostic service (1 day between tests scenario) is available.

Reducing the reporting times associated with diagnostic tests to 2 days (reported within 2 days) sees a 7 -day reduction in the pathway length for patients at both hospitals. If the reports were available the day after the test (reported within 1 day), patients at both hospitals could see between 8 and 10 days removed from their pathway.

The combined scenarios offer the largest reduction in the pathway length with between 15 and 18 days removed from the pathway if a patient is seen within 7 days and they are offered diagnostic tests (provided twice a week) and receive the results within 2 days. Between 20 and 22 days can be removed from the current lung cancer pathway if the final scenario is implemented with patients seen in clinic within 7 days and a daily diagnostic service on offer with reports available the next day.

If we combine these scenarios with a time to treatment that is limited to 21 days, we can achieve compliance with the National Optimal Lung Cancer Pathway; $95 \%$ of patients diagnosed and start treatment within 62 days. If patients are seen in clinic within 7 days, have tests that are offered twice a week and reported within 2 days, and start their treatment within 21 days, the scenario suggests that the average time in the system drops to 40 days or under for all treatment pathways in each hospital [Prince Charles Hospital: SACT (37.4 days), radiotherapy (40.1 days) and surgery (38.4 days); Royal Glamorgan Hospital: SACT (37.6 days), radiotherapy (36.6 days) and surgery (37.7 days)].

Further scenario analysis showed that with daily diagnostic tests which have next-day reporting, the time to treatment could be allowed to increase to 28 days and compliance against the national targets would still be achieved although this would add a week to the time in the system with patients spending on average 45 days on the pathway.

Table 7 shows the baseline and scenario results for the percentage of patients that spend 62 days or less on their pathway to treatment under the current system. The current target is $95 \%$. Under the current lung cancer pathway, neither hospital achieves this.

Under the first scenario (outpatients within 7 days) where patients attend an outpatient clinic within 7 days, the percentage of patients receiving their first treatment would increase by $12-16 \%$ (see Table 7). For example, under the current system, approximately $50 \%$ of surgery patients at the RGH receive their first treatment within 62 days.
However, this increases to $63 \%$ if patients can be seen in the outpatient clinic within seven days of their point of suspicion.

Under the second scenario (7 days between tests) where a weekly diagnostic service is offered, there is a slight increase in the percentage of patients that receive their treatment within 62 days at both hospitals. Patients at the Royal Glamorgan would observe a 1-2\% increase whilst those at Prince Charles see almost $7 \%$. If a daily diagnostic service ( 1 day between tests scenario) is offered, there is a larger percentage of patients receiving their treatment within 62 days; an increase of between $13 \%$ and $18 \%$.

Under the improved reporting scenarios (reported within 2 days; reported within 1 day), approximately $10 \%$ more patients would receive treatment within 62 days of their date of suspicion.

If patients could be seen in an outpatient clinic within 7 days and then be offered a daily diagnostic slot with the report available the next day, the service would see a $20 \%$ $25 \%$ increase in the number of patients starting treatment within 62 days of their date of suspicion.

Combining the bi-weekly testing scenario with a 21-day time to treatment could see almost all patients diagnosed and start their treatment within 62 days [PCH: SACT (99.5\%), radiotherapy (96.8\%), and surgery (98.8\%); RGH: SACT $(99.2 \%)$, radiotherapy (99.8\%) and surgery (99\%)]. The scenario analysis also showed that compliance could be maintained if the time to treatment was closer to 28 days provided patients were seen in clinic within 7 days, with daily diagnostic tests and the reports available the next day.

\section{Single cancer pathway}

Table 8 shows the baseline and scenario results for the single cancer pathway model if applied to each hospital.

Under the proposed single cancer pathway, the average time a patient spends on a lung cancer pathway until their decision to treat (DTT) is approximately 28 days. Under Scenario 1 where reporting is guaranteed to take place on the same day as the test, a reduction to under 20 days can be achieved.

Table 9 shows the percentage of patients that spend 28 days or less in the system under the baseline and scenario models. In the Single Cancer Pathway, lung cancer patients should receive their diagnosis within 28 days of their date of suspicion.

The baseline simulation model for the Single Cancer Pathway suggests that under the proposed single cancer 


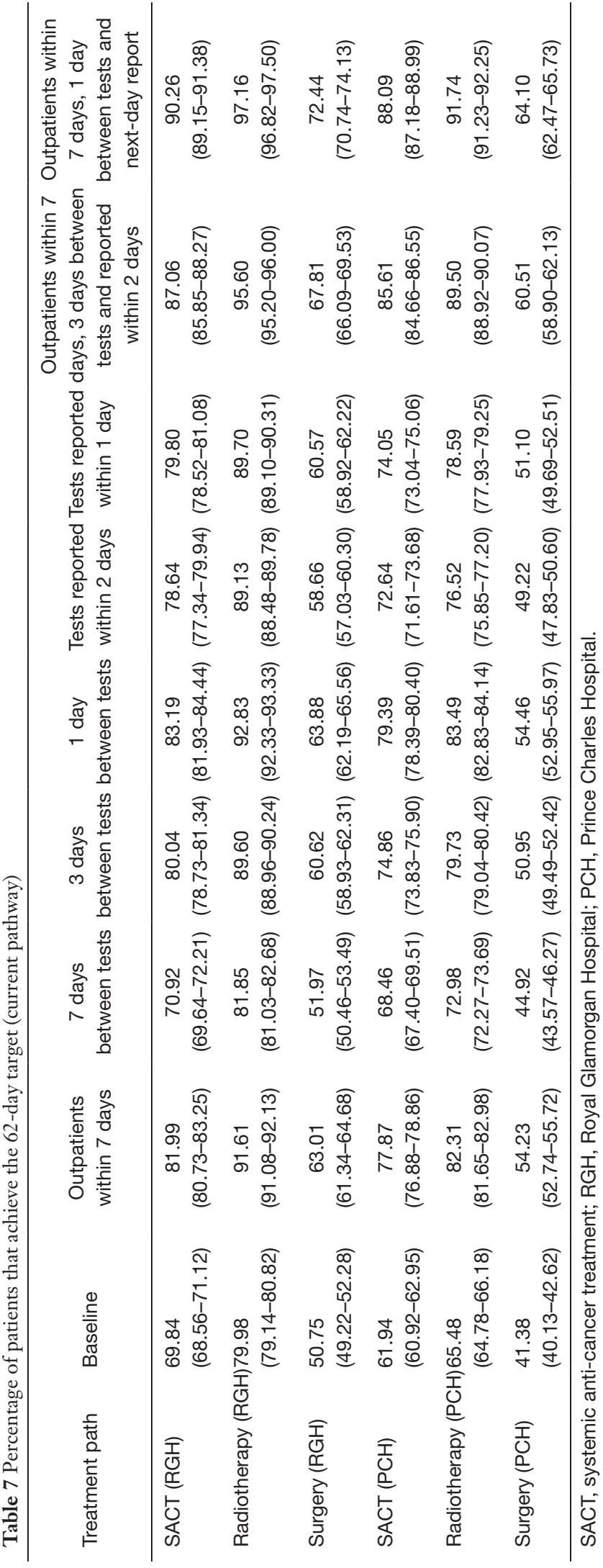

Table 8 Comparing the mean time to diagnosis on the Single Cancer Pathway (in days)

\begin{tabular}{lcc}
\hline Treatment Path & Baseline & Scenario 1 \\
\hline RGH & & \\
SACT & $27.17(26.98-27.36)$ & $19.18(19.10-19.26)$ \\
Radiotherapy & $27.00(26.73-27.26)$ & $19.27(19.16-19.37)$ \\
Surgery & $27.24(27.03-27.46)$ & $19.19(19.11-19.28)$ \\
PCH & \\
SACT & $26.44(25.91-26.97)$ & $12.06(12.00-12.12)$ \\
Radiotherapy & $26.53(26.00-27.06)$ & $12.02(11.97-12.08)$ \\
Surgery & $26.59(26.08-27.11)$ & $11.99(11.93-12.05)$ \\
\hline RGH, Royal Glamorgan Hospital; SACT, systemic anti-cancer \\
treatment; PCH, Prince Charles Hospital.
\end{tabular}

Table 9 Percentage of patients that receive a diagnosis within 28 days (SCP pathway)

\begin{tabular}{lcc}
\hline Treatment path & Baseline & Scenario 1 \\
\hline RGH & & \\
SACT & $62.00(61.23-62.77)$ & $93.45(93.06-93.84)$ \\
Radiotherapy & $61.79(60.70-62.88)$ & $93.67(93.16-94.17)$ \\
Surgery & $61.13(60.29-61.97)$ & $93.46(93.04-93.87)$ \\
PCH & & \\
SACT & $63.40(62.45-64.35)$ & 100 \\
Radiotherapy & $63.08(62.19-63.98)$ & 100 \\
Surgery & $62.44(61.51-63.38)$ & 100 \\
\hline
\end{tabular}

SCP, Single Cancer Pathway; RGH, Royal Glamorgan Hospital; SACT, systemic anti-cancer treatment; $\mathrm{PCH}$, Prince Charles Hospital.

pathway and with current resources, RGH can achieve the $95 \%$ target. With same day reporting (scenario 1), the model suggests that both hospitals could have all their patients diagnosed within 28 days of their date of suspicion.

\section{Discussion}

This study showed that discrete event simulation can be used to represent the current and proposed lung cancer pathways in two hospitals. Whilst other simulation modelling techniques are available (system dynamics, agentbased), the clinicians involved in the study were keen to examine the patient level flow through the current and 
proposed lung cancer pathways and DES lends itself to this type of problem. The DES models showed accurate representations of the system and resources currently used. Scenario analysis showed that streamlining the diagnostic tests with same- or next-day reporting and reducing the time until the initial outpatient clinic each have significant benefits in reducing the time a patient spends on the current lung cancer pathway. However, combining the scenarios sees a much-reduced time on the diagnostic pathway. Considering the simulation model for the single cancer pathway provides a useful representation of the proposed system and illustrates that patients can be diagnosed within the targeted 28 days.

Discrete event simulation modelling proved useful in providing a means of representing a complex pathway in a virtual environment which can be analysed through "what-if" scenarios. Detailed statistical analysis of the Tracker 7 lung cancer data alongside gathering expert opinion has been used to ensure the model was an accurate representation of the current system.

Typically, in developing simulation models, an important part is to build and validate the model with key personnel that work in the appropriate service. A major strength of this study was that information from respiratory consultants in each hospital was used to produce the process map that formed the structure of the simulation models. Another benefit of the simulation is that the visual representation of the current model by a series of images for each activity ensures that the process and results can be understood by clinicians, administrators, policy makers and analysts, thus making it a useful decision support tool.

Whilst DES simulation modelling allows variation to be captured in the probability distributions associated with the activities along the pathway it does not allow the user to examine an individual patient's characteristics and how they affect the time they spend on the lung cancer pathway. Therefore, a weakness in the study was that the data did not include information on the demographics or behavioural characteristics of the patients. If these had been present, we may have adapted the simulation model towards an agentbased model and used the extra information to examine the effect of a patient's characteristics on the time spent on the pathway. For example, examining the time a patient spends deciding on their diagnostic and treatment options; some patients will avoid healthcare because they do not want to know what is wrong while others will want to be seen and treated as soon as possible.

In the scenario analysis, the following areas of improvement were identified:

* Reducing the time until the patient is first seen in clinic to under 7 days

* Offering daily diagnostic tests

* Reporting the results within a day of the diagnostic test

* Reducing the time until the first treatment to 21 days

To facilitate a more streamlined diagnostic service:

* both hospitals in CTMUHB could reserve a couple of CT scan appointments, each day, for new lung cancer patient referrals and ensure that results are ready for the first outpatient clinic appointment (which should be within 7 days of the date of suspicion). Aneurin Bevan University Health Board, another health board in South Wales has already been reserving CT slots for its new referrals since 2005.

* Rapid access to subsequent investigations, with rapid reporting turnaround times, with tests done in parallel rather than sequentially.

* Radiologists would need to be available to provide same-day/next-day reports on the scans.

* There would be a need to increase the number of radiographers and radiologists providing the diagnostic tests so that the turnaround times of each test are reduced.

* The time between the treatment MDT to DTT should be a few days at most. In the Single Cancer Pathway, it is at most 3 days.

* There would also be a need to ensure that the time between the final MDT and the treatment starting is kept to a minimum with waiting lists being managed appropriately so that the waiting time does not exceed 21 days. The single cancer pathway stipulates that patients should start treatment within 21 days of their decision to treat.

However, for these improvements to happen there would need to be a substantial investment in the diagnostic workforce and infrastructure associated with the lung cancer diagnostic pathway. Further examination of the radiographers and radiologists' workload is also needed to see how daily tests and same-day/next-day reporting can be achieved in the future. Current research is considering how the capacity of diagnostics would need to change to support the single cancer pathway in Wales.

In future studies, it would be worthwhile conducting a further exercise to examine the sub-processes involved to see where further improvements can be made. For example, 
considering the steps after the decision to treat and before treatment starts. It would also be worth exploring how a patient's demographics and socio-economic background affect the decisions they make in relation to the lung cancer pathway. A recent qualitative study is looking at how people from deprived areas who are at high risk of getting lung cancer can be persuaded to ask for help earlier (22). It would be interesting to see if the findings from this study could be incorporated into future versions of the simulation model to see how a patient's demographics affect the time spent on a lung cancer pathway.

\section{Acknowledgments}

We would like to take this opportunity to thank all those staff in Cwm Taf Morgannwg University Health Board, Aneurin Bevan University Health Board, and Velindre Cancer Centre, who have supported this work through their clinical advice and supply of data. We would like to thank Sinan Eccles and Alexander Brown who provided expert knowledge of the current diagnostic pathways in their hospitals.

Funding: This work was supported by a Cancer Research UK grant 'Analysis and Modelling of a Single Cancer Pathway Diagnostics' (Early Diagnosis Project Award A27882).

\section{Footnote}

Reporting Checklist: The authors have completed the STRESS reporting checklist. Available at http://dx.doi. org/10.21037/tlcr-20-919

Data Sharing Statement: Available at http://dx.doi. org/10.21037/tlcr-20-919

Conflicts of Interest: All authors have completed the ICMJE uniform disclosure form (available at http://dx.doi. org/10.21037/tlcr-20-919). Tracey J. England, Paul R. Harper, Tom Crosby, Daniel Gartner, and Edilson F. Arruda report that they received grants from Cancer Research UK during the conduct of this study. The other authors have no conflicts of interest to declare.

Ethical Statement: The authors are accountable for all aspects of the work in ensuring that questions related to the accuracy or integrity of any part of the work are appropriately investigated and resolved. The study did not require ethical approval as no patients were directly involved and the data used to determine the model parameters was fully anonymized prior to use.

Open Access Statement: This is an Open Access article distributed in accordance with the Creative Commons Attribution-NonCommercial-NoDerivs 4.0 International License (CC BY-NC-ND 4.0), which permits the noncommercial replication and distribution of the article with the strict proviso that no changes or edits are made and the original work is properly cited (including links to both the formal publication through the relevant DOI and the license). See: https://creativecommons.org/licenses/by-nc-nd/4.0/.

\section{References}

1. Cancer Research UK, 2016 Lung Cancer Statistics. [Internet] [cited 202020 April]. Available online: https:// www.cancerresearchuk.org/health-professional/cancerstatistics/statistics-by-cancer-type/lung-cancer

2. UK Lung Cancer Coalition, 2019 Pathways Matter: A review of the National Optimal Lung Cancer Pathway.

3. United Kingdom Lung Cancer Coalition, 201625 by 25: A Ten-Year Strategy to Improve Lung Cancer Survival Rates.

4. Public Health Wales [Internet], Cancer Survival in Wales, 1995 - 2016. Available online: https://publichealthwales_ shinyapps.io/wcisu_cancer_survival_in_Wales_1995_2016

5. Lung cancer five-year net survival [Internet], Available online: https://stats.OECD.org

6. Welsh Government [Internet], 2018 Cancer Delivery Plan for the NHS to 2020. Available online: https://gov.wales/ topics/health/nhswales/plans/cancer-plan/?lang=en

7. Wales Cancer Network, 2019 National Optimal Pathway for Lung Cancer: Point of Suspicion to First Definitive Treatment in Adults (aged 16 and over).

8. Monks T, Worthington D, Allen M, et al. A modelling tool for capacity planning in acute and community stroke services. BMC Health Serv Res 2016;16:530.

9. Harper PR, Shahani AK. Modelling for the Planning and Management of Bed Capacities in Hospitals. J Oper Res Soc 2002;53:11-8.

10. Harper PR. A Framework for Operational Modelling of Hospital Resources. Health Care Manag Sci 2002;5:165-73.

11. Zhang X. Application of discrete event simulation in health care: a systematic review. BMC Health Serv Res 2018; $18: 687$.

12. Brailsford SC, Harper PR, Sykes J. Incorporating Human 
Behaviour in Simulation Models of Screening for Breast

Cancer. Eur J Oper Res 2012;291:491-507.

13. Harper PR, Jones SK. Mathematical Models for the Early Detection and Treatment of Colorectal Cancer. Health Care Manag Sci 2005;8:101-9.

14. Viana J, Brailsford SC, Harindra V, et al. Combining discrete event simulation and system dynamics in a healthcare setting: a composite model for Chlamydia infection. Eur J Oper Res 2014;237:196-206.

15. Harper PR, Shahani AK. A Decision Support System for the Care of HIV and AIDS patients in India. Eur J Oper Res 2003;147:187-97.

16. Vile JL, Gillard JW, Harper PR, et al. Time-dependent stochastic methods for managing and scheduling Emergency Medical Services. Oper Res Health Care 2016;8:42-52.

17. Tuson M, England TJ, Behrens D, et al. Modelling for the proposed roll-out of the '111' service in Wales: a case

Cite this article as: England TJ, Harper PR, Crosby T, Gartner D, Arruda EF, Foley KG, Williamson IJ. Examining the diagnostic pathway for lung cancer patients in Wales using discrete event simulation. Transl Lung Cancer Res 2021;10(3):1368-1382. doi: 10.21037/tlcr-20-919 study. Health Care Manag Sci 2018;21:159-76.

18. England T, Gartner D, Ostler E, et al. Near realtime bed modelling feasibility study. J Simul 2019. doi: 10.1080/17477778.2019.1706434.

19. Ju F, Lee HK, Osarogiagbon RU, et al. Computer modeling of lung cancer diagnosis-to-treatment process. Transl Lung Cancer Res 2015;4:404-14.

20. Monks T, Currie CSM, Onggo BS, et al. Strengthening the Reporting of Empirical Simulation Studies: introducing the STRESS guidelines. J Simul 2019;13:55-67.

21. Wales Cancer Network, 2017 Briefing Paper: Single Cancer Pathway.

22. McCutchan G, Hiscock J, Hood K, et al. Engaging highrisk groups in early lung cancer diagnosis: a qualitative study of symptom presentation and intervention preferences among the UK's most deprived communities. BMJ Open 2019;9:e025902. 